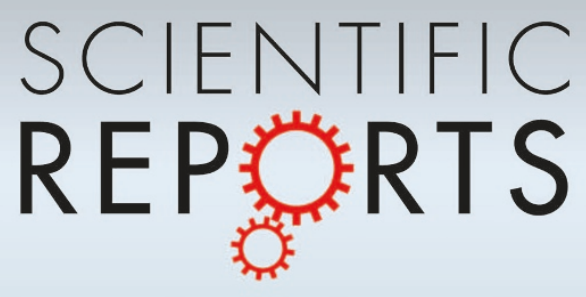

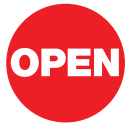

SUBJECT AREAS:

QUANTUM

INFORMATION

QUANTUM MECHANICS

QUANTUM OPTICS

INFORMATION THEORY AND COMPUTATION

Received

16 August 2012

Accepted

25 October 2012

Published

12 December 2012

Correspondence and requests for materials should be addressed to L.M. (I.mazzola@qub. ac.uk) or P.M. (paolo. mataloni@uniroma 1.it)

\section{Linear Optics Simulation of Quantum Non-Markovian Dynamics}

\author{
Andrea Chiuri ${ }^{1}$, Chiara Greganti ${ }^{1}$, Laura Mazzola ${ }^{2}$, Mauro Paternostro ${ }^{2,3}$ \& Paolo Mataloni ${ }^{1,4}$
}

'Dipartimento di Fisica, Sapienza Università di Roma, Piazzale Aldo Moro 5, I-00185 Roma, Italy, ${ }^{2}$ Centre for Theoretical Atomic, Molecular and Optical Physics, School of Mathematics and Physics, Queen's University Belfast, BT7 1NN Belfast, United Kingdom, ${ }^{3}$ Institut für Theoretische Physik, Albert-Einstein-Allee 11, Universität Ulm, D-89069 Ulm, Germany, ${ }^{4}$ Istituto Nazionale di Ottica (INO-CNR), Largo E. Fermi 6, 1-50125 Firenze, Italy.

The simulation of open quantum dynamics has recently allowed the direct investigation of the features of system-environment interaction and of their consequences on the evolution of a quantum system. Such interaction threatens the quantum properties of the system, spoiling them and causing the phenomenon of decoherence. Sometimes however a coherent exchange of information takes place between system and environment, memory effects arise and the dynamics of the system becomes non-Markovian. Here we report the experimental realisation of a non-Markovian process where system and environment are coupled through a simulated transverse Ising model. By engineering the evolution in a photonic quantum simulator, we demonstrate the role played by system-environment correlations in the emergence of memory effects.

T he simulation of quantum processes is a key goal for the grand programme aiming at grounding quantum technologies as the way to explore complex phenomena that are inaccessible through standard, classical calculators $^{1,2}$. Some interesting steps have been performed in this direction: simple condensed matter and chemical processes have been implemented on controllable quantum simulators ${ }^{3,4}$. The relativistic motion and scattering of a particle in the presence of a linear potential has been demonstrated in a trapped-ion quantum simulator ${ }^{5-7}$ that opens up the possibility to the study of quantum field theories ${ }^{8,9}$. The quantum Ising model ${ }^{10}$ has been experimentally analysed under the perspective of universal digital quantum simulators ${ }^{11}$ and very recently scaled up to hundreds of particles ${ }^{12}$.

This scenario has recently been extended to open quantum evolutions ${ }^{13}$, marking the possibility to investigate important features of the way a quantum system interacts with its environment, including the so-called sudden death of entanglement induced by a memoryless environment ${ }^{14}$.

This interaction can destroy the most genuine quantum properties of the system, or involve exchange of coherence between system and environment, giving rise to memory effects and thus making the dynamics "non-Markovian"15. Characterizing non-Markovian evolutions is currently at the centre of extensive theoretical and experimental efforts ${ }^{16-18}$. Here we demonstrate experimentally the (non-)Markovianity of a process where system and environment are coupled through a simulated transverse Ising model ${ }^{10}$ (see Supplementary Material). By engineering the evolution in a fully controlled photonic quantum simulator, we assess and demonstrate the role that system-environment correlations have in the emergence of memory effects.

The paradigmatic description of quantum open dynamics involves a physical system $S$ evolving freely according to the Hamiltonian $H^{S}$ and embedded in an environment $E$ (whose free dynamics is ruled by $H^{E}$ ). System and environment interact via the Hamiltonian $H^{S E}{ }^{15}$, which we assume to be time-independent for easiness of description. While the dynamics of the joint state $\rho_{S E}$ is closed and governed by the unitary operator $U_{t}=e^{-l\left(H^{S}+H^{E}+H^{S E}\right) t}$, the state of $S$, which is typically the only object to be accessible directly, is given by the reduced density matrix $\rho_{S}(t)=\operatorname{Tr}_{E}\left\{\rho_{S E}(t)\right\}$. For factorized initial states $\rho_{S E}(0)=\rho_{S}(0) \otimes \rho_{E}(0)$ and moving to an interaction frame defined by the free Hamiltonian of the total system, such reduced evolution can be recast in the operator-sum picture $\rho_{S}(t)=\sum_{\mu} K_{\mu}(t) \rho_{S}(0) K_{\mu}^{\dagger}$, where $\left\{K_{\mu}\right\}$ is the set of trace-preseving, non-unitary Kraus operators of $S$ that are responsible for effects such as the loss of populations and coherence from the state of the system $^{19}$.

The roots of such decoherence mechanism have long been studied, together with their intimate connection to the so-called measurement problem and the implications of the collapse of the wave function ${ }^{20-22}$. Numerous experimentally oriented techniques have been proposed to counteract decoherence ${ }^{19,23,24}$. Yet, a complementary viewpoint can be taken, where the possibility to engineering structured environments and tailored $S$-E couplings is seen as a resource to achieve longer coherence times of the system ${ }^{25}$, prepare entangled states, perform quantum 


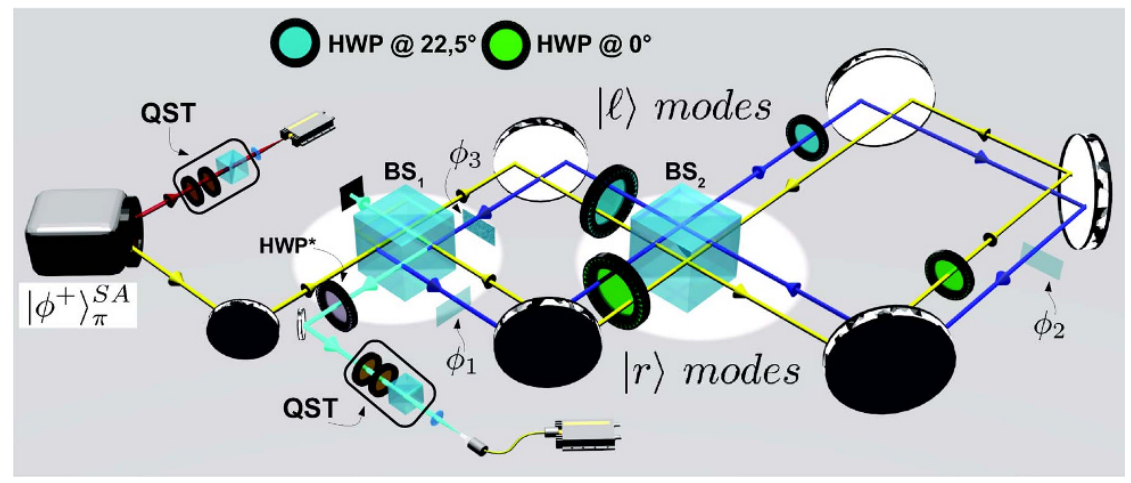

Figure $1 \mid$ The Sagnac interferometer used to perform our simulation. The red line represents the high photon, whose polarisation is immediately detected. The low photon, depicted by the yellow and blue paths, goes through the set of gates acting on its polarisation and momentum, implemented by BS, HWP, and glasses plates $\phi_{i}$. The optical axis of HWP* is kept free so as to implement the last step of the simulation. The thin glass plates $\phi_{1}, \phi_{2}$ and $\phi_{3}$ placed in the interferometer allow to set the phase of the environment evolution at each step. The Hadamard gates are implemented by setting $\phi_{1}=\phi_{2}=$ $\phi_{3}=0$ [cf. Eq. (1)].

computation, and realise quantum memories. This calls for the exploitation of memory-effects typical of a non-Markovian dynamics as a useful tool for the processing of a quantum state.

Unfortunately, a satisfactory understanding of non-Markovianity is yet to be reached, which motivates the recent and intense efforts performed towards the rigorous characterisation of non-Markovian evolutions, the formulation of criteria for the emergence of nonMarkovian features, and the proposition of factual measures for the quantification of the degree of non-Markovianity of a process ${ }^{26-29}$. Some of them have been recently used in order to characterize, both theoretically and experimentally, the character of system-environment interactions for few- and many-body quantum systems ${ }^{16-18}$. Here we use a photonic setup to simulate a system-environment coupling ruled by a transverse Ising spin model (see Supplementary Material). Such interaction gives rise to the non-Markovian evolution of $S$, as witnessed by the non-monotonic behavior of its entanglement with an ancilla $A$ that is shielded from the environmental effects. Our goal is the experimental investigation of the fundamental connection between system-environment correlations and non-Markovianity. The simulator that we propose allows for the implementation of various free evolutions of $S$ and $E$, as well as the adjustment of their mutual coupling, thus making possible the transition from deeply non-Markovian dynamics, all the way down to a fully forgetful regime.

\section{Results}

Experimental setup and theoretical model. The simulator is experimentally realised using different degrees of freedom of the photonic device shown in Fig. 1, which consists of the concatenation of two Mach-Zehnder interferometers and a single Sagnac loop. The information carriers are two photons (referred hereafter as "high" and "low"): the system $S$ is embodied by the polarisation of the low photon (which can be either horizontal $|H\rangle \equiv|0\rangle$ or vertical $|V\rangle \equiv|1\rangle$ ), while the environment $E$ is encoded in the longitudinal momentum degree of freedom (the path) of the same photon (which will be right $|r\rangle \equiv|0\rangle$ or left $|l\rangle \equiv|1\rangle)$. The ancilla $A$ is embodied by the polarisation of the high photon. High and low photons are emitted by the source of polarization-entangled described in the Methods.

The ancilla is the key tool for our goals. Indeed, to investigate the emergence of non-Markovianity in the evolution of $S$ due to its interaction with $E$, we use the method proposed in Ref. 27: we focus on the modifications induced by the $S$ - $E$ coupling on a prepared entangled state of $S$ and $A$. If the $S$ - $A$ entanglement decays monotonically in time, the dynamics of $S$ is fully Markovian. Differently, if for certain time-windows there is a kick-back from $E$ that makes such entanglement increase, the dynamics is necessarily non-Markovian.
In fact, if the local action of the environment is no longer represented by a continuous family of completely positive maps, the $S$ - $A$ entanglement is no longer constrained to decrease monotonically. This is evidence of the flow-back of coherence on the system and results in an increment of the $S-A$ entanglement.

As in other digital quantum simulators, the dynamics is approximated by a stroboscopic sequence of quantum gates. Conceptually, the simulation consists of the forward evolution of $S$ over discrete time slices $^{30}$ according to a Trotter-Suzuki decomposition ${ }^{31}$ of the total time propagator (see Supplementary Material). This approach is known to be effective for quantum simulation ${ }^{7}$ and is implemented here by the sequence of operations shown in Fig. 2a). The free evolution of the environment is accounted for by the Hadamard gate $\mathcal{H}^{E}=\left(\sigma_{z}^{E}+\sigma_{x}^{E}\right) / \sqrt{2}\left(\sigma_{m}^{j}\right.$ is the $m=x, y, z$ Pauli matrix of qubit $j=S, E$ and $\mathcal{H}^{j}$ is the Hadamard gate of qubit $\left.j=S, E\right)$, while $H^{S} \propto \sigma_{z}^{S}$. The $S$ - $E$ interaction is engineered by implementing the controlled-rotation $\quad G^{E S}=|0\rangle\left\langle\left. 0\right|_{E} \otimes \mathbb{1}^{S}+\mid 1\right\rangle\left\langle\left. 1\right|_{E} \otimes \mathcal{R}^{S}(\varphi)\right.$, which rotates the system according to the general single-qubit operation $\mathcal{R}^{S}(\varphi)=\cos \varphi \mathbb{1}^{S}-\imath \sin \varphi \sigma_{y}^{S}$ depending on the state of the environment. This class of conditional operations is obtained from Hamiltonian generators of the two-qubit transverse Ising form (see Supplementary Material), which motivates our choice and is thus the class of system-environment interactions that is simulated in this work. Experimentally, we fix $\varphi=\pi / 4$ so as to implement a conditional- $\left(\sigma_{x}^{S} \mathcal{H}^{S}\right)$ gate. By using the identity $\sigma_{z}^{S}=\mathcal{H}^{S} \sigma_{x}^{S} \mathcal{H}^{S}$, it is straightforward to prove that this gate is locally equivalent (via $\sigma_{z}^{S}$ ) to the composition of a controlled Hadamard and a controlled anti- $Z$ (i.e. a gate applying $\sigma_{z}^{S}$ only when $E$ is in $|0\rangle$ ), namely $\mathcal{G}^{E S} \equiv C \mathcal{H}_{k \pi} \overline{C Z}_{k \pi}=|0\rangle\left\langle\left. 0\right|_{E} \otimes \sigma_{z}^{S}+\mid 1\right\rangle\left\langle\left. 1\right|_{E} \otimes \mathcal{H}^{S}\right.$. The extra $\sigma_{z}^{S}$ needed to make the two conditional gates equivalent can thus be absorbed in $H^{S}$, which thus becomes $\mathbb{1}^{S}$.

This set of gates is experimentally realised in our photonic simulator as sketched in Fig. 2b). The $\mathcal{H}^{E}$ gate is implemented by means of a beam splitter (BS) in conditions of temporal and spatial indistinguishability of the optical modes. This scheme allows to evolve the input modes $|r\rangle$ and $|\ell\rangle$ at each step as

$$
|r\rangle \rightarrow \frac{1}{\sqrt{2}}\left(|r\rangle+e^{i \phi i}|\ell\rangle\right), \quad|\ell\rangle \rightarrow \frac{1}{\sqrt{2}}\left(|r\rangle-e^{i \phi i}|\ell\rangle\right),
$$

where the phases $\phi_{i}$ are varied by rotating thin glass plates intercepting one of the optical modes entering the BS. In order to perform a more general rotation of $E$ it will be necessary to unbalance the output modes (using intensity attenuators) making the probability of occurrence of $|r\rangle$ and $|\ell\rangle$ unequal.

The controlled gate $C \mathcal{H}_{k \pi}$ is realised by placing a half-wave plate (HWP) on one of the two output modes with optical axis at $22.5^{\circ}$ 

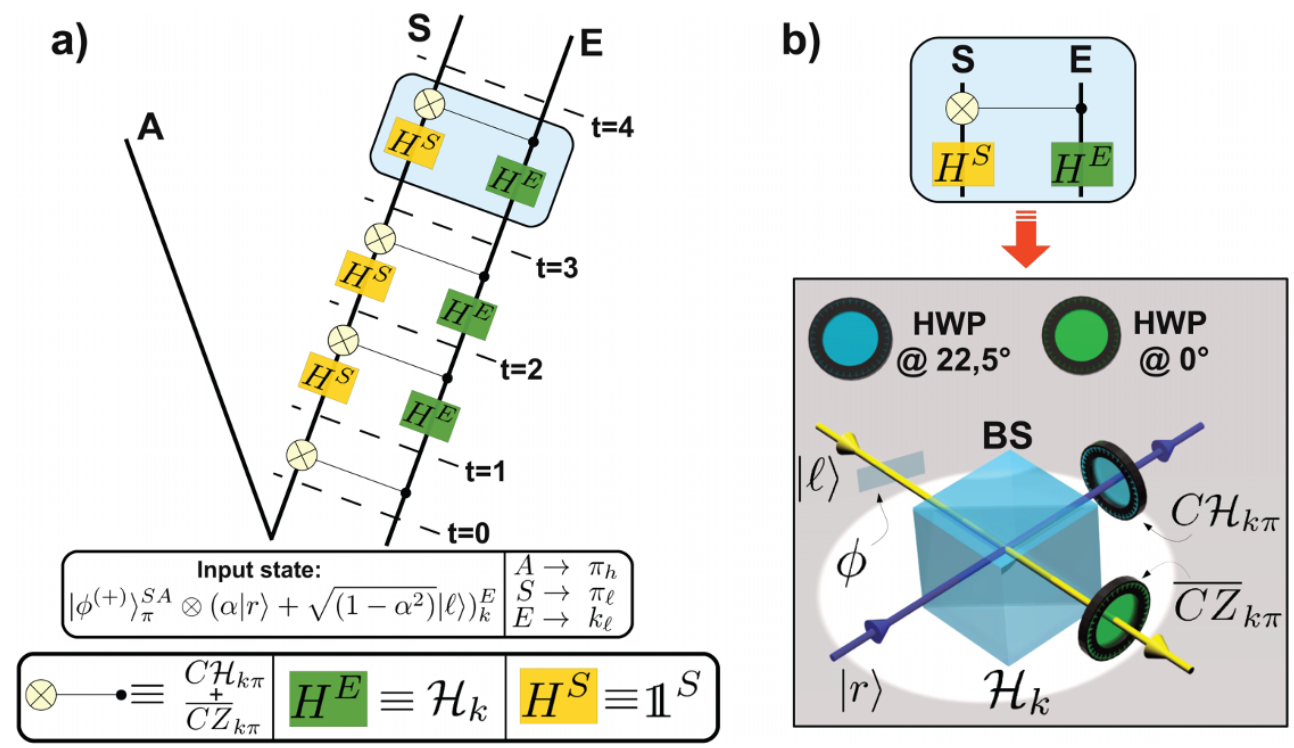

Figure $2 \mid$ Scheme of principle of the simulation. (a) A maximally entangled state of $A$ and $S$ is prepared. $S$ and $E$ then evolve through the application of free single-qubit gates $\left(H^{E}\right.$ and $\left.H^{S}\right)$ and joint two-qubits $\left(C \mathcal{H}_{k \pi} \overline{C Z}_{k \pi}\right)$ ones. Time increases from bottom to top of the figure. Here $\pi_{h(\ell)}$ stands for the polarization of the high (low) photon, while $k_{\ell}$ for the momentum of the low photon. (b) Each block of the scheme is technically realised by the concatenation of a BS and two HWPs oriented at different angles.

with respect to the vertical direction. The temporal delay introduced by this waveplate is compensated by another HWP on the opposite output mode with optical axis set at $0^{\circ}$ with respect to the vertical direction. This implements the $\overline{C Z}$ gate, as shown in Fig. 2b). In order to assess non-Markovianity, ancilla and system are prepared in the maximally entangled state $\left|\phi^{+}\right\rangle_{\pi}^{S A}=(|H H\rangle+|V V\rangle) / \sqrt{2}$. This twoqubit Bell state is engineered using the polarization entanglement source of Ref. 32 (see Methods). The environment is initialised in $|\chi\rangle_{k}^{E}=\left(\alpha|r\rangle+\sqrt{1-\alpha^{2}}|\ell\rangle\right)_{k}^{E}\left(\alpha \in \mathbb{R}\right.$ is set by the transmittivity of $B S_{1}$ [cf. Fig. 1] and can be varied by placing an intensity attenuator on one of the two output modes), a state endowed with quantum coherence as it is key due to the conditional nature of the dynamics that we simulate. The stability, modular structure and long coherence time of our interferometric setting allows for the repeated iteration of each block of gates $\mathcal{G}^{E S}\left(\mathcal{H}^{E} \otimes \mathbb{1}^{S}\right)$.

As $A$ does not evolve with the environment, the polarisation state of the high photon (depicted in red in Fig. 1) is immediately detected and only the low photon (in yellow in Fig. 1) goes through each sequence of gates at the various steps. A standard optical setup for the performance of quantum state tomography $(\mathrm{QST})^{33}$ is used to reconstruct the state of the $S$ - $A$ system after the evolution. The radiation is collected by using an integrated system composed of GRaded INdex (GRIN) lens and single-mode fibre ${ }^{34}$, and is then detected by single-photon counters. For each step of the simulated dynamics, we measure the state of the environment by projecting the polarisation qubits on the states $|H H\rangle,|H V\rangle,|V H\rangle,|V V\rangle$. Finally, we trace out the degrees of freedom of the $S$ - $A$ system by summing up the corresponding counts measured for every single projection needed for the implementation of single-qubit QST. The Pauli operators for $E$, generated after the first passage through the $B S_{1}$, are measured using $B S_{2}$. The same procedure is followed to perform the QST of the environmental state at each step. The state of the $S$ - $A$ system is reconstructed in a similar way, by summing up the counts collected after projecting $E$ onto $|r\rangle$ and $|\ell\rangle$.

Non-Markovian simulation. Figure 3 summarises the results obtained by running through the evolution of the overall system. In order to quantify entanglement we use the entanglement of formation $\operatorname{EOF}(\mathrm{SA})^{35}$ between $S$ and $A$, which is operatively linked to the cost of engineering a given state by means of Bell-state resources (see the Methods section). We are also interested in the correlations shared by the environment with the rest of the system, hence we evaluate the von Neumann entropy of the environment, defined as $S(E)=-\operatorname{Tr}\left[\rho_{E} \log _{2}\left(\rho_{E}\right)\right]$, which under the assumption of pure total ASE state quantifies the entanglement in the partition AS against $\mathrm{E}$. Figure 3a) shows the experimental entanglement of formation at each time step (black-square points) against the results of a theoretical model (red line) that, including all the most relevant sources of imperfections, deviates from the ideal picture sketched above. First, the BSs are not entirely polarisation-insensitive: for $B S_{1}$ and $\mathrm{H}(\mathrm{V})$ polarisation the reflectivity over transmittivity ratio $R / T=42 / 58$ (45/ 55), while for $B S_{2}$ is $R / T=45 / 55$ (55/45). Second, although the desired input state $\left|\phi^{+}\right\rangle_{\pi}^{S A}$ is created with high fidelity $(\simeq 93 \%$, see Supplementary Material), the entangled-state source generates spurious $|H V\rangle$ and $|V H\rangle$ components, accounting for about $5 \%$ of the total state, which reduce the initial system-environment entanglement to about 0.8 . The inclusion of such imperfections makes the agreement between theory and experimental data very good up to the fourth step of our simulation, showing at least one revival of the $S$ - $A$ entanglement and thus witnessing the non-Markovian nature of the evolution ${ }^{27}$. The fifth experimental point is significantly far from the theoretical behaviour because of the not-ideal setting of the phases $\phi_{i}$ $(i=1,2,3)$. In fact, we have verified that their values are not completely polarization independent. This determines a slight difference for the four contributions $|H H\rangle_{S A},|H V\rangle_{S A},|V V\rangle_{S A},|V H\rangle_{S A}$ entering the state. This imperfection affects the performance of each Hadamard gate and becomes significant especially for the last step, where the cumulative effect of three $\mathcal{H}^{E}$ gates should be considered. Furthermore, the fifth point is affected by an uncompensated phase shift induced by the multilayer mirrors used in our setting [cf. Figure 1], as explained in the Supplementary Information. Nonetheless, this point still reveals successfully the occurrence of a second entanglement revival, thus strengthening our conclusions.

Figure 3b) compares the experimentally inferred EOF(SA) (black squares) to the von Neumann entropy of the environment $S(E)$ (green circles) quantifying the correlations shared between $E$ and $S$ - $A$. These figures of merit appear to be perfectly anti-correlated, thus giving evidence of a trade-off between the amount of entanglement that $S$ and $A$ can share at the expenses of $S$ - $E$ correlations. This 
a)

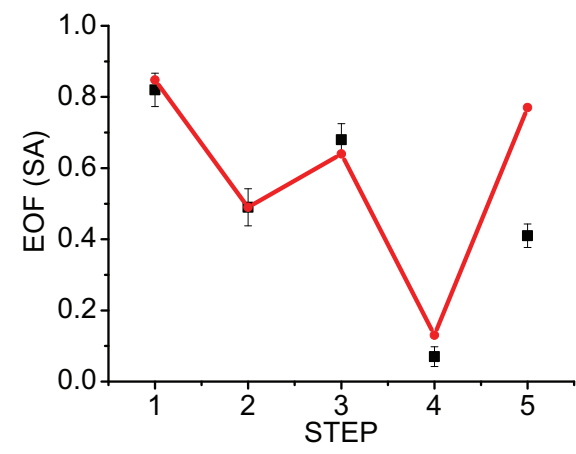

b)

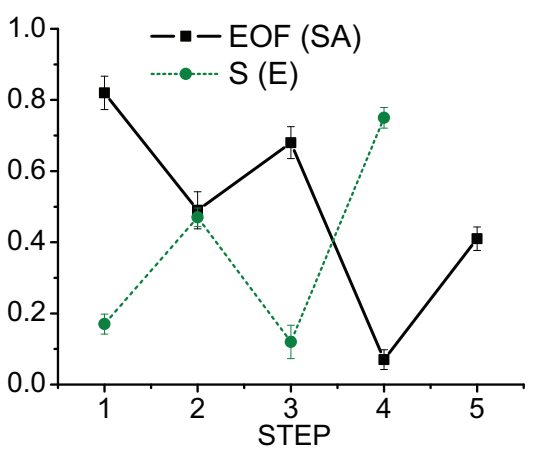

c)

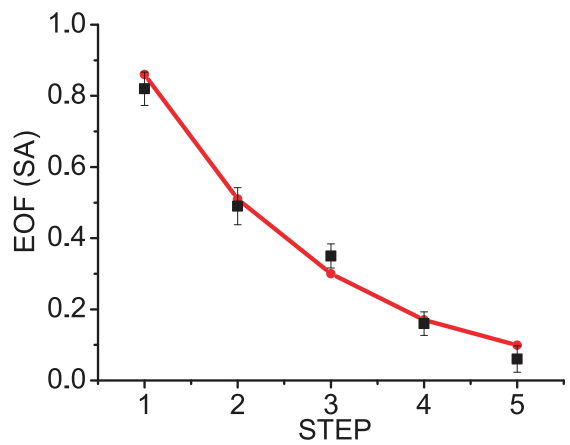

Figure 3 Results of the simulation of the (non-)Markovian regimes. (a) Theoretical prediction (red line) against experimentally inferred data (black squares) for EOF(SA) as against the steps of the non-Markovian simulation. Two revivals of EOF(SA) are clearly visible. (b) Comparison between the experimental evolution of $\mathrm{EOF}(\mathrm{SA})$ (black squares) and $\mathrm{S}(\mathrm{E})$ (green circles) representing the correlations between $E$ and $S$ - $A$. The two evolutions are clearly anti-correlated. In order to measure the Pauli operators needed to reconstruct the state of $E$ at the fifth step, a further BS will be necessary. (c) Theoretical behaviour (red line) and experimental data (black squares) for EOF(SA) in the Markovian simulation.

strengthens the idea that correlations with the environment play a fundamental role in this process, a point that has been addressed in Ref. 36 where it is shown that the establishment of system-environment correlations is a necessary conditions for the emergence of non-Markovianity. This result can be bridged with our analysis considering that the mixed system-environment state of Ref. 36 can be purified by enlarging the Hilbert space of the system including an appropriate ancilla. The anti-correlation between $\mathrm{S}(\mathrm{E})$ and $\mathrm{EOF}(\mathrm{SA})$ suggest interesting connections with the monogamous nature of quantum entanglement in such tripartite system ${ }^{37}$. We have illustrated such connections in the Supplementary Information, where a theoretical simulation for up to 11 steps is reported and a quantitative analysis fof the entanglement sharing features in our system is provided.

To reinforce our point even further, we now explore the Markovian counterpart of our simulation by replacing the unitary evolution of $E$ with an incoherent map that resets the environment into the very same state at each step of the evolution. As the key role in the $S$-E interaction is played by quantum coherence, we chose to re-set the environment into a completely mixed state. This is realised by spoiling the temporal indistinguishability of the optical modes entering the BS by mutually delaying the $|r\rangle$ and $|\ell\rangle$ components. Intuitively, by making the state of the environment rigid, we wash out any possibility for system-environment correlations, thus pushing the dynamics towards Markovianity. This intuition is fully confirmed by the experimental evidences collected via QST: the black squares in Figure 3c) show a monotonic (quasi-exponential) decay of EOF(SA) (matching our theoretical predictions, red line), which is in perfect agreement with the absence of $S-E$ quantum correlations as signalled by the positivity of the partially transposed $S-E$ state.

\section{Discussion}

By simulating a non-trivial two-qubit coupling model, we have demonstrated the non-Markovianity of the evolution induced on $S$ by a dynamical environment and a system-environment interaction allowing for kick-back of coherence. By adopting a witness that makes use of the effects that non-Markovianity has on entanglement, we have explored experimentally the link between the emergence of non-Markovianity and system-environment correlations. The next step in this endeavour will be the experimental proof that nonMarkovianity can be used as a resource for the advantageous processing of information ${ }^{38,39}$, such as the preparation of interesting states ${ }^{40}$, along the lines of previous studies on state engineering and information manipulation through Markovian processes ${ }^{41-43}$. Our setup will be particularly well suited for this task, in light of the effective control over both system and environment that can be engineered both in space (thanks to the modular nature of the setting) and in time.

\section{Methods}

Source of entangled states. The black box shown in Fig. 1 of the main text represents the source used for the generation of the hyperentangled state encoded in the polarization and path degrees of freedom of the photons produced by spontaneous parametric down-conversion. Here polarization entanglement, corresponding to the state $\left|\phi^{+}\right\rangle=\frac{1}{\sqrt{2}}(|H H\rangle+|V V\rangle)$, was realized by spatial and temporal superposition of photon pair emissions occurring with equal probability, back and forth, from a single barium borate (BBO) type-I crystal under double excitation of a vertically polarized UV CW pump beam. A suitable rotation of the polarization on one of the two possible emission directions of the photons was then applied. The source that we have used is such that the two photons, belonging to the degenerate $\mathrm{BBO}$ emission cone, are also path entangled, as explained in Ref. 32. For our pourposes we need to select a pair of correlated directions, belonging to the emission cone surface, along which two photons travel, obtaining in this way the high and low photons shown in red and yellow in Fig. 1.

Entanglement of formation. For two qubits, the entanglement of formation is given by $^{35} \mathrm{EOF}=h\left(\frac{1+\sqrt{1-C^{2}}}{2}\right)$, with $h(x)=-x \log _{2} x-(1-x) \log _{2}(1-x)$ and $C=$ $\max \left\{0, \lambda_{1}-\lambda_{2}-\lambda_{3}-\lambda_{4}\right\}$. Here, $\left\{\lambda_{i}\right\}$ is the set of eigenvalues (arranged in nonincreasing order) of the Hermitian matrix $R=\sqrt{\sqrt{\rho} \tilde{\rho} \sqrt{\rho}}$ where $\tilde{\rho}=\left(\sigma_{y} \otimes \sigma_{y}\right) \rho^{*}\left(\sigma_{y} \otimes \sigma_{y}\right)$ and $\rho^{*}$ is the conjugate of the density matrix.

1. Buluta, I. \& Nori, F. Quantum simulators. Science 326, 108111 (2009).

2. Cirac, J. I. \& Zoller, P. Goals and opportunities in quantum simulation. Nature Phys. 8, 264 (2012).

3. Ma, X.-S., Dakic, B., Naylor, W., Zeilinger, A. \& Walther, P. Quantum simulation of the wavefunction to probe frustrated Heisenberg spin systems. Nature Phys. 7, 399 (2011).

4. Lanyon, B. P. et al. Towards quantum chemistry on a quantum computer. Nature Chem. 2, 106 (2010).

5. Gerritsma, R. et al. Quantum simulation of the Dirac equation. Nature 463, 68 (2010).

6. Gerritsma, R. et al. Quantum Simulation of the Klein Paradox with Trapped Ions. Phys. Rev. Lett. 106, 060503 (2011).

7. Blatt, R. \& Roos, C. F. Quantum simulations with trapped ions. Nature Phys. 8, 277 (2012).

8. Casanova, J. et al. Quantum Simulation of Quantum Field Theories in Trapped Ions. Phys. Rev. Lett. 107, 260501 (2011).

9. Casanova, J., Mezzacapo, A., Lamata, L. \& Solano, E. Quantum Simulation of Interacting Fermion Lattice Models in Trapped Ions. Phys. Rev. Lett. 108, 190502 (2012).

10. Sachdev, S. Quantum Phase Transitions (Cambridge Univ. Press, 2011).

11. Lanyon, B. P. et al. Universal Digital Quantum Simulation with Trapped Ions. Science 334, 6052 (2011).

12. Britton, J. W. et al. Engineered two-dimensional Ising interactions in a trappedion quantum simulator with hundreds of spins. Nature 484, 489 (2012). 
13. Barreiro, J. T. et al. An open-system quantum simulator with trapped ions. Nature 470, 486 (2011).

14. Almeida, M. P. et al. Environment-Induced Sudden Death of Entanglement Science 316, 579 (2007).

15. Breuer, H.-P. \& Petruccione, F. The Theory of Open Quantum Systems (Oxford Univ. Press, 2007).

16. Liu, B.-H. et al. Experimental control of the transition from Markovian to nonMarkovian dynamics of open quantum systems. Nature Phys. 7, 931 (2011).

17. Liu, B.-H. et al. Photonic realization of nonlocal memory effects and nonMarkovian quantum probes, arXiv:1208.1358

18. Apollaro, T. J. G. et al. Memory-keeping effects and forgetfulness in the dynamics of a qubit coupled to a spin chain. Phys. Rev. A 83, 032103 (2011).

19. Nielsen, M. A. \& Chuang, I. L. Quantum Computation and Quantum Information (Cambridge Univ. Press, 2000).

20. Zurek, W. H. Decoherence and the transition from quantum to classical. Phys. Today 44, 36 (1991).

21. Zurek, W. H. Decoherence, einselection, and the quantum origins of the classical. Rev. Mod. Phys. 75, 715 (2003).

22. Schlosshauer, M. Decoherence, the measurement problem, and interpretations of quantum mechanics. Rev. Mod. Phys. 76, 1267 (2005).

23. Zanardi, P. \& Rasetti, M. Noiseless Quantum Codes. Phys. Rev. Lett. 79, 3306 (1997).

24. Viola, L. \& Lloyd, S. Dynamical suppression of decoherence in two-state quantum systems. Phys. Rev. A 58, 2733 (1998).

25. Myatt, C. J. et al. Decoherence of quantum superpositions through coupling to engineered reservoirs. Nature 403, 269 (2000).

26. Wolf, M. M., Eisert, J., Cubitt, T. S. \& Cirac, J. I. Assessing Non-Markovian Quantum Dynamics. Phys. Rev. Lett. 101, 150402 (2008).

27. Rivas, Á., Huelga, S. F. \& Plenio, M. B. Entanglement and non-Markovianity of quantum evolutions. Phys. Rev. Lett. 105, 050403 (2010).

28. Breuer, H.-P., Laine, E.-M. \& Piilo, J. Measure for the degree of non-Markovian behavior of quantum processes in open systems. Phys. Rev. Lett. 103, 210401 (2009).

29. Lu, X.-M., Wang, X. \& Sun, C. P. Quantum Fisher Information Flow in Non-Markovian Processes of Open Systems. Phys. Rev. A 82, 042103 (2010).

30. Lloyd, S. Universal Quantum Simulators. Science 273, 1073 (1996).

31. Trotter, H. F. On the product of semi-groups of operators. Proc. Am. Math. Soc. 10, 545 (1959).

32. Barbieri, M. et al. Polarization-momentum hyperentangled states: Realization and characterization. Phys. Rev. A 72, 052110 (2005).

33. James, D., Kwiat, P., Munro, W. \& White, A. Measurement of qubits. Phys. Rev. A 64, 052312 (2001).

34. Rossi, A., Vallone, G., Chiuri, A., De Martini, F. \& Mataloni, P. Multipath entanglement of two photons. Phys. Rev. Lett. 102, 153902 (2009).

35. Wootters, W. K. Phys. Rev. Lett. 80, 2245 (1998).
36. Mazzola, L., Rodriguez-Rosario, C., Modi, K. \& Paternostro, M. Dynamical role of system-environment correlations in non-Markovian dynamics. Phys. Rev. A 86, 010102(R) (2012)

37. Coffman, V., Kundu, J. \& Wootters, W. K. Phys. Rev. A 61, 052306 (2000)

38. Plenio, M. B. \& Huelga, S. F. Dephasing assisted transport: Quantum networks and biomolecules. J. Chem. Phys. 131, 105106 (2009).

39. Caruso, F., Chin, A. W., Datta, A., Huelga, S. F. \& Plenio, M. B. Highly efficient energy excitation transfer in light-harvesting complexes: The fundamental role of noise-assisted transport. J. Chem. Phys. 131, 105106 (2009).

40. Huelga, S. F., Rivas, A. \& Plenio, M. B. Non-Markovian assisted Steady State Entanglement. Phys. Rev. Lett. 108, 160402 (2012).

41. Plenio, M. B. \& Huelga, S. F. Entangled light from white noise. Phys. Rev. Lett. 88, 197901 (2002).

42. Kraus, B. et al. Preparation of Entangled States by Quantum Markov Processes. Phys. Rev. A 78, 042307 (2008).

43. Verstraete, F., Wolf, M. M. \& Cirac, J. I. Quantum computation, quantum state engineering, and quantum phase transitions driven by dissipation. Nature Phys. $\mathbf{5}$, 633 (2009).

\section{Acknowledgments}

This work was supported by EU-Project CHISTERA-QUASAR, PRIN 2009 and FIRB-Futuro in ricerca HYTEQ, the EU under a Marie Curie IEF Fellowship (L.M.), and the UK EPSRC (M.P.) under a Career Acceleration Fellowship and a grant of the "New Directions for Research Leaders" initiative (EP/G004579/1).

\section{Author contributions}

M.P. proposed the original idea, A.C. and P.M. designed the experimental setup. A.C., C.G. and P.M. performed the experiment, L.M. and M.P. analysed and interpreted the data. All authors contributed to the writing of the manuscript.

\section{Additional information}

Supplementary information accompanies this paper at http://www.nature.com/ scientificreports

Competing financial interests: The authors declare no competing financial interests.

License: This work is licensed under a Creative Commons

Attribution-NonCommercial-NoDerivs 3.0 Unported License. To view a copy of this license, visit http://creativecommons.org/licenses/by-nc-nd/3.0/

How to cite this article: Chiuri, A., Greganti, C., Mazzola, L., Paternostro, M. \& Mataloni, P. Linear Optics Simulation of Quantum Non-Markovian Dynamics. Sci. Rep. 2, 968 DOI:10.1038/srep00968 (2012). 